\title{
Atypical Clinical Presentation of Geriatric Syndrome in Elderly Patients With Pneumonia or Coronary Artery Disease
}

\author{
Yu Jin Jung, Jong Lull Yoon², Hak Sun Kim², Ae-Young Lee ${ }^{1}$, Mee Young Kim², Jung Jin Cho \\ ${ }^{1}$ Department of Family Medicine, Hallym University Dongtan Sacred Heart Hospital, Hwaseong, ${ }^{2}$ Department of Family Medicine, Hallym \\ University College of Medicine, Chuncheon, Korea
}

Corresponding Author: Jong Lull Yoon, MD, PhD Department of Family Medicine, Hallym University Dongtan Sacred Heart Hospital, Hallym University College of Medicine, 7 Keunjaebong-gil, Hwaseong 18450, Korea

Tel: $+82-31-8086-2370$ Fax: +82-31-8086-2242 E-mail: lull@hallym.ac.kr

Received: August 1, 2017 Revised: October 26, 2017 Accepted: October 26, 2017

\begin{abstract}
Background: Atypical symptoms often occur in elderly patients due to impaired homeostasis associated with age-related physiological changes and multiple pathologies. These atypical symptoms make diagnosis difficult and may partially increase morbidity and mortality. This study aimed to determine the incidence of atypical clinical presentation and to identify the effects of age and comorbidities on illness presentation in the elderly. Methods: Medical charts of 6,057 elderly patients ( $\geq 60$ years) with pneumonia or coronary artery disease (CAD) admitted to 4 university hospitals were retrospectively reviewed. Determinants of atypical symptom presentation was evaluated using logistic regression analysis. The definition of atypical presentation was adapted from a previous study on atypical symptoms of pneumonia and CAD. Results: Among the 6,057 participants, $4,773(78.8 \%)$ and 1,284 (21.2\%) presented with typical and atypical symptoms, respectively. Among the participants, $24.8 \%$ CAD and $18.8 \%$ pneumonia patients had atypical presentations. Logistic regression analysis showed that factors associated with atypical presentation in CAD patients were age ( $\geq 85$ years; odds ratio [OR], $2.7 ; 95 \%$ confidence interval [CI], 1.81-4.03), higher pulse rate $(\mathrm{OR}, 1.01 ; 95 \% \mathrm{Cl}, 1.00-1.01)$, and number of comorbidities $\geq 4$ (OR, 1.62; 95\% $\mathrm{Cl}, 1.13-2.32)$. In pneumonia patients, age ( $\geq 85$ years; $\mathrm{OR}, 2.22 ; 95 \% \mathrm{Cl}, 1.49-3.31$ ), body mass index (OR, 0.97; $95 \% \mathrm{Cl}, 0.94-0.99)$, and 1 comorbidity (OR, 1.53; $95 \% \mathrm{Cl}, 1.01-2.36)$ were statistically significant factors that increased atypical presentation. Conclusion: This study suggested that older patients frequently present atypical geriatric syndrome with acute illness, and age and comorbidity are statistically significant factors associated with atypical symptoms in CAD or pneumonia patients.
\end{abstract}

Key Words: Geriatric syndrome, Atypical symptom, Older, Pneumonia, Coronary artery disease

\section{INTRODUCTION}

The world's population is continuously aging as the average lifespan increases. According to recent statistical data, the elderly population is expected to approximately 1.6 billion people worldwide in 2050. Korea has especially the fastest aging population worldwide. Therefore, a more comprehensive and meticulous approach to improve healthy life expectancy of older patients vulnerable to multiple chronic disorders should be implemented.

Many elderly persons have simultaneously occurring chronic degenerative disorders. Because of these characteristics of geriatric disorders, typical symptoms of illnesses are often absent in elderly patients, and they often present with atypical symptoms such as confusion, fall, general weakness, malaise, incontinence, and immobility, commonly known as "geriatric syndrome"1-5). Previous studies have reported atypical illness presentation associated with aging ${ }^{6-11)}$. These symptoms are more common in frail older persons and can be markers of frailty in elderly people ${ }^{12)}$. Some older patients may present with minimal symptoms that are disproportionate to the severity of their illness, and others may complain symptoms related to an organ system but far from the primarily affected organ ${ }^{13)}$. According to several studies, atypical presentations were found in approximately $20 \%$ of elderly patients in the Emergency Department (ED), and only 20\%-30\% of geriatric patients with infection presented with fever at the ED ${ }^{14,15)}$. This ambiguous presentation of symptoms results in difficult diagnosis of pneumonia or myocardial infarction as well as exacerbations of chronic diseases, such as depression". Timely recognition of atypical symptoms of acute disease states can help avoid diagnostic errors and delayed treatments. Five common diseases with atypical presentations in older 
patients may include infectious disease, cardiovascular disease, acute abdomen, hyperthyroidism, and depression ${ }^{16)}$. In this study, we assessed the incidence of atypical symptoms in patients with pneumonia and cardiovascular diseases and examined whether nonspecific symptoms in the elderly increase with age and whether the number of underlying diseases is associated with the presentation of atypical symptoms.

\section{MATERIALS AND METHODS}

\section{Participants}

The study was conducted at 4 university hospitals in Korea between January 2014 and December 2016. We retrospectively reviewed the medical records of all patients aged $\geq 60$ years diagnosed with coronary artery disease (CAD) or pneumonia and admitted to the Departments of Family Medicine and Internal Medicine during the study period. Main symptoms were assessed on the first day of hospitalization, such as vital signs and laboratory results, and data on underlying diseases were identified in the discharge records. Myocardial infarction and angina were classified as CAD. The Korean Standard Classification Version 6 was used as a standardized format for the discharge diagnostic code (angina, 1201-129; myocardial infarction, I210-1219; pneumonia, J120-J189, J851).

During the study period, 10,680 cases were found in the 4 hospital's medical records. Cases with missing or inadequate information on symptom presentation were excluded. Patients who were hospitalized for chemotherapy or a scheduled cardiac procedure were also excluded. For patients hospitalized several times during the study period, all visits were included. After excluding 4,623 cases, a total 6,057 were included in this study. The protocol of this study was approved by the Institutional Review Board of the Hallym University Dongtan Sacred Heart Hospital (approval number: 2017-04-201-001).

\section{Definition of Atypical Symptoms}

The participants were divided into 2 groups based on their chief complaints: typical and atypical symptom. The dominant symptom was assumed to be presented as the chief complaint and included with other accompanying symptoms on the admission record.

According to a previous study, atypical presentation was defined as the lack of typical symptoms expected for a final diagnosis or unusual symptoms unrelated to the final diagnosis $^{17)}$.

Similar to the previous study ${ }^{2)}$, typical symptoms in pneumonia were fever/chill, cough, sputum, rhinorrhea, sore throat, dyspnea, and chest pain. Based on the study by Then et al. ${ }^{18)}$, the typical symptoms of CAD were chest pain/discomfort/tightness, epigastric pain, and radiating pain to the arm, shoulder, and neck.

If patients presented any of the typical symptoms, they were included in the typical symptom group. If the chief complaint was atypical, but accompanied with other symptoms included in the typical symptoms, the patient was included in the atypical group.

\section{Statistical Analysis}

To estimate the age and comorbidity effects, both groups were further stratified into four age groups ( $<65$ years, $65-74$ years, $75-84$ years, and $\geq 85$ years). Based on the associations between symptom presentation and age, a number of comorbidities were also explored using the correlation analysis. The t-test was used to compare the differences in body mass index (BMI), vital signs, and laboratory tests (hemoglobin, cholesterol, protein, and albumin) between the 2 symptom groups. A logistic regression model was developed using variables with $p$-values $<0.05$ in the univariate analysis, which was considered a statistically significant difference between the groups. All analyses were conducted using IBM SPSS Statistics ver. 24.0 (IBM Co., Armonk, NY, USA).

\section{RESULTS}

Table 1 shows the demographic data of all 6,057 patients. A total of $4,773(78.8 \%)$ and 1,284 patients (21.2\%) presented with typical and atypical symptoms, respectively. Among the participants, $24.8 \%$ of CAD and $18.8 \%$ pneumonia patients had atypical presentations. Both $C A D$ and pneumonia patients presenting atypical symptoms were older than the typical symptom group. Based on the correlation test, Pearson correlation coefficients in CAD patients are 0.18 (age) and 0.17 (number of comorbidity), whereas those in pneumonia patients are 0.14 (age) and 0.02 (number of comorbidity). Age, sex, pulse, diabetes mellitus (DM), and the number of comorbidities affected the presentation of atypical symptoms in CAD. In pneumonia patients, age and BMI were statistically significant factors associated with atypical symptoms.

Table 2 shows the dominant atypical symptoms. Dyspnea (50.2\%), general weakness $(9.8 \%)$, dizziness $(9.2 \%)$, syncope (6.3\%), mental changes (4.5\%), and nausea (2.0\%) were observed in CAD patients, while general weakness (27.3\%), mental changes $(25.4 \%)$, poor oral intake $(6.5 \%)$, hemoptysis (5.7\%), and myalgia (4.4\%) were observed in pneumonia patients.

Table 3 shows the adjusted logistic regression model developed using variables with $p$-values of $<0.05$. We included possible factors and comorbidities in the logistic regression model, although the $p$-value was not significant in pneumonia. Determinants associated with atypical symptom presentation in CAD patients were age $(65-74,75-84, \geq 85$ years), 
Yu Jin Jung, et al.

Table 1. Baseline characteristic of patients based on symptom presentation

\begin{tabular}{|c|c|c|c|c|c|c|}
\hline \multirow{2}{*}{ Variable } & \multicolumn{3}{|c|}{$\mathrm{CAD}(\mathrm{n}=2,427)$} & \multicolumn{3}{|c|}{ Pneumonia $(n=3,630)$} \\
\hline & Typical & Atypical & $\mathrm{p}$-value & Typical & Atypical & $\mathrm{p}$-value \\
\hline No. of patients & $1,826(75.2)$ & $601(24.8)$ & & $2,947(81.2)$ & $683(18.8)$ & \\
\hline Age (yr) & $72.3 \pm 7.8$ & $75.6 \pm 8.1$ & $0.001^{*}$ & $76.5 \pm 8.7$ & $78.6 \pm 8.4$ & $0.001^{*}$ \\
\hline$<65$ & $381(85.8)$ & $63(14.2)$ & & $318(87.6)$ & $44(12.4)$ & \\
\hline $65-74$ & $741(79.3)$ & $194(20.7)$ & & $906(84.8)$ & $160(15.2)$ & \\
\hline $75-84$ & $562(67.6)$ & $269(32.4)$ & & $1,170(78.6)$ & $317(21.4)$ & \\
\hline$\geq 85$ & $142(7.8)$ & $75(34.6)$ & & $553(77.2)$ & $162(22.8)$ & \\
\hline Sex & & & $0.001^{*}$ & & & 0.482 \\
\hline Female & $953(52.2)$ & $268(44.6)$ & & $1,615(54.8)$ & $364(53.3)$ & \\
\hline Male & $873(47.8)$ & $333(55.4)$ & & $1,332(45.2)$ & $319(46.7)$ & \\
\hline BMI $\left(\mathrm{kg} / \mathrm{m}^{2}\right)$ & $24.6 \pm 3.4$ & $24.6 \pm 4.0$ & 0.971 & $22.4 \pm 4.1$ & $21.7 \pm 3.9$ & $<0.001^{*}$ \\
\hline $\mathrm{SBP}(\mathrm{mmHg})$ & $133.5 \pm 30.4$ & $132.9 \pm 46.8$ & 0.760 & $134.1 \pm 28.4$ & $134.9 \pm 22.1$ & 0.390 \\
\hline DBP (mmHg) & $81.2 \pm 32.7$ & $80.4 \pm 31.6$ & 0.966 & $80.9 \pm 21.9$ & $81.3 \pm 13.4$ & 0.586 \\
\hline Pulse (/min) & $81.0 \pm 16.6$ & $85.02 \pm 35.3$ & $<0.001^{*}$ & $134.1 \pm 28.4$ & $134.9 \pm 22.1$ & 0.541 \\
\hline $\mathrm{Hb}(\mathrm{g} / \mathrm{dL})$ & $12.1 \pm 1.7$ & $12.0 \pm 1.8$ & 0.113 & $11.4 \pm 1.8$ & $11.2 \pm 1.9$ & 0.080 \\
\hline Cholesterol (mg/dL) & $139.9 \pm 43.2$ & $137.6 \pm 41.2$ & 0.270 & $120.7 \pm 42.1$ & $122.1 \pm 45.5$ & 0.457 \\
\hline Albumin (g/dL) & $3.7 \pm 0.6$ & $3.6 \pm 0.6$ & 0.380 & $3.3 \pm 0.6$ & $3.2 \pm 0.5$ & 0.530 \\
\hline Protein $(\mathrm{g} / \mathrm{dL})$ & $6.5 \pm 0.8$ & $6.4 \pm 0.9$ & 0.166 & $6.4 \pm 1.2$ & $6.4 \pm 1.4$ & 0.380 \\
\hline No. of comorbidity & $3.1 \pm 2.5$ & $4.1 \pm 2.8$ & $<0.001^{*}$ & $4.0 \pm 3.1$ & $4.3 \pm 3.2$ & 0.457 \\
\hline 0 & $163(76.9)$ & $49(23.1)$ & & $251(86.0)$ & $41(14.0)$ & \\
\hline 1 & 348 (83.9) & $67(16.1)$ & & $409(79.4)$ & $106(20.6)$ & \\
\hline 2 & 406 (81.2) & $94(18.8)$ & & $463(82.7)$ & $97(17.3)$ & \\
\hline 3 & $325(83.3)$ & 65 (16.7) & & $422(79.6)$ & $108(20.4)$ & \\
\hline$\geq 4$ & $584(64.2)$ & $326(35.8)$ & & $1,402(80.9)$ & $331(19.1)$ & \\
\hline $\mathrm{DM}$ & $391(21.4)$ & $168(27.0)$ & $0.001^{*}$ & $644(21.9)$ & $169(24.7)$ & 0.100 \\
\hline Dyslipidemia & $110(6.0)$ & $37(6.2)$ & 0.900 & $175(5.9)$ & $42(6.1)$ & 0.828 \\
\hline Cancer & $3,993(21.5)$ & $141(23.5)$ & 0.323 & $697(23.7)$ & 177 (25.9) & 0.213 \\
\hline Dementia & $59(3.2)$ & $17(2.8)$ & 0.618 & $125(4.2)$ & $30(4.4)$ & 0.855 \\
\hline Stroke & $29(1.6)$ & $12(2.0)$ & 0.500 & $43(1.5)$ & $16(2.3)$ & 0.103 \\
\hline
\end{tabular}

Values are presented as number (\%) or mean \pm standard deviation.

$\mathrm{CAD}$, coronary artery disease; BMI, body mass index; SBP, systolicc blood pressure; DBP, diastolic blood pressure; Hb, hemoglobin; DM, diabetes mellitus.

"p-values obtained by correlation test, Student t-test.

pulse, and comorbidities of $\geq 4$. Less than three comorbidities tended to decrease the presentation of atypical symptoms in CAD patients. Age ( $75-84, \geq 85$ years), BMl, and the presence of one comorbidity were statistically significant factors that increased the likelihood of atypical presentation in pneumonia.

\section{DISCUSSION}

In this study, the rates and types of atypical symptom observed were similar to those in previous studies. Our study is the first to evaluate the effects of the number of comorbidities. Elderly patients often present with several comorbidities, which increases the likelihood of adverse drug reactions to multidrug administration and induces more complex problems than basic functional deterioration due to aging. In this regard, we examined the effects of multiple comorbidities on symptoms and the relationship between some underlying diseases and atypical symptoms.

Treatment of the altered expressions of illness and formulation of health policy in the oldest elderly - in severity, presentation, and perception - are clinically challenging ${ }^{19)}$. Previous studies have shown that when classic ischemic precordial pain is present, elderly patients with myocardial infarction are less likely to complain of pain ${ }^{20,21)}$ and have asymptomatic symptom ratios ranging from $4.7 \%$ to $33 \%$ with poorer clinical outcomes ${ }^{17,22-24)}$. Dyspnea, nausea, vomiting, cough, palpitations, and anxiety have been reported. In most studies, dyspnea is the most common among the atypical symptoms, accounting for $26 \%-49 \%$ of all cases ${ }^{9,25)}$. 
Table 2. Prevalences of atypical symptoms according to diseases

\begin{tabular}{|c|c|}
\hline Coronary artery disease & No. (\%) \\
\hline Dyspnea & $302(50.2)$ \\
\hline General weakness & $59(9.8)$ \\
\hline Dizziness & $55(9.2)$ \\
\hline Syncope & $38(6.3)$ \\
\hline Mental change & $27(4.5)$ \\
\hline Nausea & $12(2)$ \\
\hline Abdominal pain & $11(1.8)$ \\
\hline Headache & $9(1.5)$ \\
\hline Palpitation & $9(1.5)$ \\
\hline Back pain & $8(1.3)$ \\
\hline Myalgia & $8(1.3)$ \\
\hline Edema & $6(1)$ \\
\hline Poor oral intake & $6(1)$ \\
\hline Vomiting & $6(1)$ \\
\hline Leg edema & $5(0.8)$ \\
\hline \multicolumn{2}{|l|}{ Pneumonia } \\
\hline General weakness & $188(27.3)$ \\
\hline Mental change & $175(25.4)$ \\
\hline Poor oral intake & $45(6.5)$ \\
\hline Dizziness & $40(5.8)$ \\
\hline Hemoptysis & $39(5.7)$ \\
\hline Myalgia & $30(4.4)$ \\
\hline Syncope & $26(3.8)$ \\
\hline Abdominal pain & $20(2.9)$ \\
\hline Vomiting & $17(2.5)$ \\
\hline Nausea & $16(2.3)$ \\
\hline Headache & $13(1.9)$ \\
\hline Back pain & $9(1.3)$ \\
\hline Febrile sense & $8(1.2)$ \\
\hline Anorexia & $5(0.7)$ \\
\hline Oliguria & $4(0.6)$ \\
\hline
\end{tabular}

Values are based on chief complaint on medical record.

Age is a well-known risk factor for CAD, with $30 \%$ of acute myocardial infarction occurring in patients aged $\geq 75$ years and $>60 \%$ of patients hospitalized for unstable angina who are $\geq 65$ years $^{26)}$. In a previous study of acute coronary syndrome (ACS), patients with atypical symptoms (atypical pain or dyspnea) were older and had more cardiovascular risk factors than those with typical chest pain ${ }^{25)}$.

A study of both ACS-suspected and ACS-diagnosed inpatients demonstrated similar prevalence and showed a statistically significant increase in atypical symptoms for 3 age groups (18-64, 65-74, $\geq 75$ years). DM and dementia are often associated with atypical symptoms for $C A D^{25-27)}$. DM has been considered the biggest factor related to atypical symptom of CAD, whereas several studies found no such association ${ }^{27-30)}$. In patients with diabetes, atypical symptoms such as dyspnea,
Table 3. Multiple logistic regression analysis on the relationship between determinants and symptom presentation

\begin{tabular}{|c|c|c|}
\hline Variable & OR $(95 \% \mathrm{CI})$ & p-value \\
\hline \multicolumn{3}{|l|}{ CAD } \\
\hline \multicolumn{3}{|l|}{ Age (yr) } \\
\hline$<65$ & 1 & \\
\hline $65-74$ & $1.47(1.07-2.02)$ & $0.017^{*}$ \\
\hline $75-84$ & $2.57(1.88-3.51)$ & $0.001^{*}$ \\
\hline$\geq 85$ & $2.7(1.81-4.03)$ & $0.001^{*}$ \\
\hline \multicolumn{3}{|l|}{ Sex } \\
\hline Male & 1 & \\
\hline Female & $0.88(0.72-1.07)$ & 0.196 \\
\hline Pulse & $1.01(1.00-1.01)$ & $0.023 *$ \\
\hline \multicolumn{3}{|l|}{ Comorbidity } \\
\hline DM (-) & 1 & \\
\hline $\mathrm{DM}(+)$ & $1.18(0.94-1.48)$ & 0.154 \\
\hline \multicolumn{3}{|c|}{ No. of comorbidity } \\
\hline \multicolumn{3}{|l|}{0} \\
\hline 1 & $0.62(0.41-0.95)$ & $0.033 *$ \\
\hline 2 & $0.75(0.50-1.11)$ & 0.144 \\
\hline 3 & $0.6(0.39-0.92)$ & $0.020^{*}$ \\
\hline 4 & $1.62(1.13-2.32)$ & $0.009 *$ \\
\hline \multicolumn{3}{|l|}{ Pneumonia } \\
\hline BMI $\left(\mathrm{kg} / \mathrm{m}^{2}\right)$ & $0.97(0.94-0.99)$ & $0.005 *$ \\
\hline \multicolumn{3}{|l|}{ Age (yr) } \\
\hline$<65$ & 1 & \\
\hline $65-74$ & $1.24(0.83-1.84)$ & 0.291 \\
\hline $75-84$ & $1.79(1.23-2.61)$ & $0.002 *$ \\
\hline$\geq 85$ & $2.22(1.49-3.31)$ & $<0.001^{*}$ \\
\hline \multicolumn{3}{|c|}{ No. of comorbidity } \\
\hline 0 & 1 & \\
\hline 1 & $1.53(1.01-2.36)$ & $0.051^{*}$ \\
\hline 2 & $1.15(0.75-1.78)$ & 0.521 \\
\hline 3 & $1.34(0.87-2.06)$ & 0.187 \\
\hline 4 & $1.3(0.88-1.91)$ & 0.194 \\
\hline
\end{tabular}

Multivariate analysis was performed using the forward stepwise variable selection procedure. The p-value of the Hosmer-Lemeshow goodness-of-fit test for final logistic regression model: coronary artery disease, 0.114 , pneumonia, 0.959 .

$\mathrm{OR}$, odds ratio; $\mathrm{CI}$, confidence interval; $\mathrm{CAD}$, coronary artery disease; DM, diabetes mellitus; BMI, body mass index.

"p-values obtained by multivariate logistic regression analyses.

confusion, nausea, and fatigue may be the presenting complaint in approximately $40 \%$ of acute myocardial infarction ${ }^{31,32)}$. Many studies have reported a correlation in diabetes patients between silent ischemia and the presence of either parasympathetic cardiac or peripheral sympathetic autonomic neuropathy ${ }^{29)}$. According to Zellweger et al. ${ }^{33,34)}$, diabetes patients represented $50 \%$ of the atypical presentation group, and this subset of the population was associated with higher mor- 
tality rates, particularly when dyspnea was the principal presentation. However, this study showed that DM did not affect the presentation of atypical symptoms when adjusted logistic regression was applied to other variables, such as age and sex. Our data lacked information on autonomic neuropathy; therefore, further investigation on the effects of DM is needed.

In the present study, we did not study the treatment and complications of CAD. Previous studies have shown that patients without chest pain were more likely to have a history of hypertension, DM, or heart failure ${ }^{13,17)}$. If their symptoms were atypical, patients with STEMI were less likely to receive $\beta$-blocker, aspirin, fibrinolysis, or primary percutaneous coronary intervention. In addition, hospital complications of heart failure, arrhythmias, and renal failure were common in patients with atypical presentation.

Regardless of the type of comorbidity, four or more comorbidities increased the likelihood of atypical symptom presentation by 1.6 times compared with CAD patients without underlying diseases. In patients with less than 3 comorbidities, atypical symptoms were fewer; however, the exact reason for this decrease could be identified.

Aging neurons may decrease the peripheral sensitivity, causing reduced pain sensation ${ }^{2}$. Additionally, atypical symptoms result from a combination of age-related physiological changes and chronic diseases.

Many review articles on patients with pneunomia include limited research on the relationship between nonspecific symptoms and age, and few studies have compared atypical symptoms for different age groups. Limpawattanaa et al. $^{15)}$ analyzed the prevalence and risk factors of atypical presentation for various infectious diseases in patients aged $\geq 65$ years who visited the ED. Thirty-five percent of patients with infectious diseases presented atypical symptoms, and pneumonia was the most common (30\%). Urinary tract infections, cancer, dementia, and pulse rate were identified as risk factors; however, their association with the number of underlying diseases was not studied. With regard to the number of medications, the difference was insignificant between the 2 groups. Atypical presentation and the number of medications were not associated. Wester et al. ${ }^{4)}$ evaluated patients with bacteremia caused by Escherichia coli and Streptococcus pneumoniae divided into 3 age groups. This study reported that the 2 oldest age groups ( $\geq 65$ and $\geq 85$ years) more frequently presented with atypical symptoms and had comorbid illnesses compared with the median and interquartile ranges. However, our study showed that age and the number of comorbidities affect the prevalence of atypical symptoms.

In the previously mentioned study, we examined whether each of the 10 common underlying diseases was related to atypical symptom presentation of the elderly. As a result, 2 diseases, i.e., cancer and dementia, showed a significant increased atypical symptoms $^{14,15)}$. In this study, factors on 5 specific diseases (DM, dyslipidemia, cancer, dementia, and stroke) were insignificant. In addition, compared to the type of disease, comorbidities had an increased atypical symptom presentation by interacting with each other. However, in pneumonia patients, only one comorbidity showed significant results, and unlike our expectation, comorbidity is not an interaction but a specific disease that may be a significant factor. Future research involving a larger number of underlying diseases is needed.

In older patients, the lack of a febrile response to infection may be due to problems with maintaining body temperature, immune system dysfunction especially in cell-mediated immunity, hypothalamus' diminished sensitivity to pyrogens, or failure to produce and conserve body heat ${ }^{3,13)}$. With increasing age, organ systems lose their ability to compensate for challenges to normal homeostasis induced by injuries to other organs. Additionally, atypical presentations may be the endproduct of idiosyncratic, complex interactions between cognitive and functional impairment, multiple comorbidities, and communication problems with acute illness or an exacerbation of a chronic disease in facing age-related physiological changes $^{13,14)}$.

As a retrospective study, one of its major limitations is that information related to atypical symptoms was extracted from only chief complaints in medical records; hence, researchers should perform a second-hand interpretation. However, the nature of data was almost invariably well documented in the medical charts. Therefore, misinterpretations were unlikely. Another limitation is that we were unable to examine the role of other factors, such as alcohol consumption, smoking, lifestyle, education, socioeconomic status, religiousness, or other medications.

In patients aged $\geq 75$ years, atypical presentation is common and should be anticipated. The absence of typical symptoms may delay diagnosis and increase morbidity and mortality. Thus, physicians should be cautious when dealing with vague symptoms in CAD patients aged $\geq 65$ years or those with comorbidity, as well as in pneumonia patients aged $\geq 75$ years and those with comorbidity. Additionally, clinicians should educate elderly patients not to underestimate mild symptoms or unpleasant sensations.

Conflicts of Interest Disclosures: The researchers claim no conflicts of interest.

\section{REFERENCES}

1. Cho B, Son KY, Oh B, Kim SJ, Kwon IS, Park BJ, et al. Development and validity and reliability of Korean comprehensive assessment tools for geriatric ambulatory care. J Korean Geriatr Soc 2013;17:18-27.

2. Johnson JC, Jayadevappa R, Baccash PD, Taylor L. Nonspecific 
presentation of pneumonia in hospitalized older people: age effect or dementia? J Am Geriatr Soc 2000;48:1316-20.

3. Yoshikawa TT. Epidemiology and unique aspects of aging and infectious diseases. Clin Infect Dis 2000;30:931-3.

4. Wester AL, Dunlop O, Melby KK, Dahle UR, Wyller TB. Agerelated differences in symptoms, diagnosis and prognosis of bacteremia. BMC Infect Dis 2013;13:346.

5. Yahav D, Schlesinger A, Daitch V, Akayzen Y, Farbman L, AbuGhanem Y, et al. Presentation of infection in older patients: a prospective study. Ann Med 2015;47:354-8.

6. Canto JG, Rogers WJ, Goldberg RJ, Peterson ED, Wenger NK, Vaccarino V, et al. Association of age and sex with myocardial infarction symptom presentation and in-hospital mortality. JAMA 2012;307:813-22.

7. Fried LP, Storer DJ, King DE, Lodder F. Diagnosis of illness presentation in the elderly. J Am Geriatr Soc 1991;39:117-23.

8. Leventhal EA, Prohaska TR. Age, symptom interpretation, and health behavior. J Am Geriatr Soc 1986;34:185-91.

9. Bayer AJ, Chadha JS, Farag RR, Pathy MS. Changing presentation of myocardial infarction with increasing old age. J Am Geriatr Soc 1986;34:263-6.

10. Williams ME. Clinical implications of aging physiology. Am J Med 1984;76:1049-54.

11. Samiy AH. Clinical manifestations of disease in the elderly. Med Clin North Am 1983;67:333-44.

12. Jarrett PG, Rockwood K, Carver D, Stolee P, Cosway S. Illness presentation in elderly patients. Arch Intern Med 1995;155: 1060-4.

13. Emmett KR. Nonspecific and atypical presentation of disease in the older patient. Geriatrics 1998;53:50-2,58-60.

14. Rutschmann OT, Chevalley T, Zumwald C, Luthy C, Vermeulen B, Sarasin FP. Pitfalls in the emergency department triage of frail elderly patients without specific complaints. Swiss Med Wkly 2005;135:145-50.

15. Limpawattanaa $P$, Mitsungnern T, Phungoen $P$, Tansangworn N, Laosuangkoon W. A secondary analysis of atypical presentations of older patients with infection in the emergency department of a tertiary care hospital in Thailand. Asian Biomed 2017;10:181-7.

16. Ham RJ, Sloane PD. Primary care geriatrics: a case-based approach. St. Louis (MO): Mosby Year Book; 1992.

17. Brieger D, Eagle KA, Goodman SG, Steg PG, Budaj A, White $\mathrm{K}$, et al. Acute coronary syndromes without chest pain, an underdiagnosed and undertreated high-risk group: insights from the Global Registry of Acute Coronary Events. Chest 2004;126: 461-9.

18. Then KL, Rankin JA, Fofonoff DA. Atypical presentation of acute myocardial infarction in 3 age groups. Heart Lung 2001; 30:285-93.

19. Minaker KL, Rowe J. Health and disease among the oldest old: a clinical perspective. Milbank Mem Fund Q Health Soc 1985;
63:324-49.

20. Culic V, Eterovic D, Miric D, Silic N. Symptom presentation of acute myocardial infarction: influence of sex, age, and risk factors. Am Heart J 2002;144:1012-7.

21. Gregoratos G. Clinical manifestations of acute myocardial infarction in older patients. Am J Geriatr Cardiol 2001;10:345-7.

22. Canto JG, Shlipak MG, Rogers WJ, Malmgren JA, Frederick PD, Lambrew CT, et al. Prevalence, clinical characteristics, and mortality among patients with myocardial infarction presenting without chest pain. JAMA 2000;283:3223-9.

23. Lusiani L, Perrone A, Pesavento R, Conte G. Prevalence, clinical features, and acute course of atypical myocardial infarction. Angiology 1994;45:49-55.

24. Uretsky BF, Farquhar DS, Berezin AF, Hood WB Jr. Symptomatic myocardial infarction without chest pain: prevalence and clinical course. Am J Cardiol 1977;40:498-503.

25. El-Menyar A, Zubaid M, Sulaiman K, AlMahmeed W, Singh R, Alsheikh-Ali AA, et al. Atypical presentation of acute coronary syndrome: a significant independent predictor of in-hospital mortality. J Cardiol 2011;57:165-71.

26. Samaras N, Chevalley T, Samaras D, Gold G. Older patients in the emergency department: a review. Ann Emerg Med 2010; 56:261-9.

27. Acharya DU, Shekhar YC, Aggarwal A, Anand IS. Lack of pain during myocardial infarction in diabetics--is autonomic dysfunction responsible? Am J Cardiol 1991;68:793-6.

28. Ochiai ME, Lopes NH, Buzo CG, Pierri H. Atypical manifestation of myocardial ischemia in the elderly. Arq Bras Cardiol 2014;102:e31-3.

29. Sniderman A, Michel C, Racine N. Heart disease in patients with diabetes mellitus. J Clin Epidemiol 1992;45:1357-70.

30. Callaham PR, Froelicher VF, Klein J, Risch M, Dubach P, Friis R. Exercise-induced silent ischemia: age, diabetes mellitus, previous myocardial infarction and prognosis. J Am Coll Cardiol 1989;14:1175-80.

31. Berry C, Tardif JC, Bourassa MG. Coronary heart disease in patients with diabetes: part I: recent advances in prevention and noninvasive management. J Am Coll Cardiol 2007;49:63142.

32. Jacoby RM, Nesto RW. Acute myocardial infarction in the diabetic patient: pathophysiology, clinical course and prognosis. J Am Coll Cardiol 1992;20:736-44.

33. Arslanian-Engoren C, Engoren M. Physiological and anatomical bases for sex differences in pain and nausea as presenting symptoms of acute coronary syndromes. Heart Lung 2010;39:386-93.

34. Zellweger MJ, Hachamovitch R, Kang X, Hayes SW, Friedman JD, Germano G, et al. Prognostic relevance of symptoms versus objective evidence of coronary artery disease in diabetic patients. Eur Heart J 2004;25:543-50. 